\title{
A Novel Watermarking Algorithm for Color Images Based on Discrete Wavelet Transform
}

\author{
Anubhav Kumar, Member, IACSIT, and Anuradha
}

\begin{abstract}
It is very easy to copy and distributed the digital data illegally. There are many methods for solving this issue like intellectual, material right protection and Copy right protection for authors, owners, distributors. As the advancement, invisible double digital watermarking technique has received considerable attention. This paper gives a robust digital watermarking algorithm for color images based on discrete wavelet transform. Here, we choose a suitable method to watermarking algorithm for color images based on Haar discrete wavelet transform. The experiment result are very well, clearly represent the transparence and robustness of the algorithm. The experiment result shown that this algorithm is also secure to various attack.
\end{abstract}

Index Terms-Watermarking, Haar wavelet, DWT, IDWT, PSNR, NC.

\section{INTRODUCTION}

Digital watermarking technology is basically known for the practical importance in the intellectual property rights. The forthcoming technology has become more advanced and fast development by the use of digital watermarking. In the recent years the digital watermarking has achieved result in the field of correlation-based techniques, frequency domain techniques, DCT based techniques, DFT based techniques and DWT based techniques. Digital watermarking is a technique of embedding some information into the given data, which is extracted later or being detected for various purposes The data embedded is called as watermark and the given data is original information. Song Qiang and Zhang Hongbin [1] proposed a new color image watermarking scheme based on image self-embedding techniques. Song Qiang and Zhang Hongbin techniques based on the bilinear interpolation and DWT). In the recent tool in the field watermarking is Singular value decomposition. Chih-Chin Lai and Cheng-Chih Tsai [2] are proposed a hybrid image watermarking scheme based on discrete wavelet transform (DWT) and singular value decomposition (SVDTwo-Level DCT and SVD watermarking for copyright protection and authenticity is proposed by Jian Yu Bai and Feng Liu [3]. Most of the domain transformation watermarking schemes works with DWT and DCT [4].

Digital watermarking scheme for color image based on image watermarking scheme for color image based on image

Manuscript received January 5, 2014; revised March 8, 2014.

Anubhav Kumar is with the Raj Kumar Goel Institute of Technology for Women, Ghaziabad, India (e-mail: rajput.anubhav@gmail.com).

Anuradha is with the Electronics and Communication Engineering Department, Laxmi Devi Institute of Engineering and Technology, Alwar, India (e-mail: anuradhamit@gmail.com). fusion using wavelet transform based on R, G, B color space is proposed by Zhou Zude et al. [5]. A novel DMWT algorithm of DCIW is proposed by Shujiao Liao, which focus on the original RGB color space and used it.

G. Sun and Y. Yu [6] proposed a digital watermarking embedding algorithm based on discrete wavelet transform (DWT) for color image. The original color image is transformed into the YIQ color space, and the watermark was embedded in both $\mathrm{Y}$ component and Q component. P. S. Huang et al. [7] presents an approach for hiding the watermark into DC components of the colour image directly in the spatial domain, followed by a saturation adjustment technique performed in RGB space.

D. Kundur and D. Hatzinakos [8] presents a novel robust watermarking approach called FuseMark based on the principles of image fusion for copy protection or robust tagging applications and C. S. Lu and H. Y. M. Liao [9] proposed a novel multipurpose watermarking scheme, in which robust and fragile watermarks are simultaneously embedded, for copyright protection and content authentication. Watermarking using discrete multiwavelet transform proposed, where L. Ghouti et al. [10] presents a robust watermarking algorithm using balanced multiwavelet transform and P. Kumswat et al. [11] proposed the spread spectrum image watermarking algorithm using the discrete multiwavelet transform.

The operation course of our technique only involves Haar discrete wavelet transform and Inverse Haar discrete wavelet transform to the R, G, B elements of color original image and watermark image respectively. The reminder of this paper is organized as follows: In Section II, the proposed scheme, including the watermark generation, embedding, and extraction is introduced. In Section III, the experimental results and analysis are presented to show the effectiveness of this scheme. Conclusions are finally drawn in Section IV.

\section{PROPOSED AlgORITHM}

\section{A. Watermark Embedding Algorithm}

The procedure of the watermarking generation and embedding process is described in Fig. 1. Step for embedding algorithm steps are as follows:

Step 1: In our proposed algorithm first of all watermark image and the original color image both are resolved to three colors RGB channels.

Step 2: After first step, DWT is taken for each layers of original and watermark image. Further two levels Haar discrete wavelets transform is used. In Fig. 2, the indexing of subbands in two level wavelet decomposition is described. 


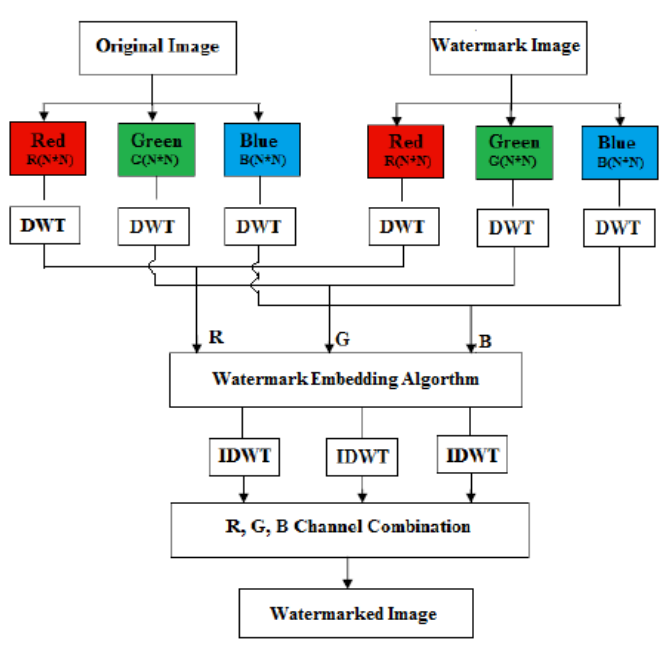

Fig. 1. Watermark embedding algorithm block diagram.



Fig. 2. Two-level wavelet decomposition.

Step 3: After discrete wavelet transform, the watermark information is embedded in to the original image and a two dimensional image is obtained. A good visual quality is achieved using a different coefficient for embedding the watermark information. This algorithm shows that the original color digital image is decomposed into three color components of $O(r), O(g), O(b)$, respectively, resulting in three color components of two layer of wavelet decomposition.

Step 4: The decomposed color components obtain are $W(r)$, $W(g), W(b)$ respectively for same color digital watermark. The decomposition coefficients corresponding to this original color image obtained by embedding the digital watermark of three primary color (RGB) used are in the formula given below [12]:

$$
\begin{aligned}
& O(r) A(i, j)=O(r) A(i, j)+\alpha_{1} w(r) A(i, j) \\
& O(g) A(i, j)=O(g) A(i, j)+\alpha_{2} w(g) A(i, j) \\
& O(b) A(i, j)=O(b) A(i, j)+\alpha_{3} w(b) A(i, j)
\end{aligned}
$$

The parameter $\alpha 1, \alpha 2, \alpha 3$ are called $\mathrm{R}, \mathrm{G}, \mathrm{B}$ embedding intensity. Where $\mathrm{A}(i, j)$ is the original image of the red, green, blue, component. These affect the validity of the algorithm directly.

Step 5: Reconstruction the processed image use the inverse discrete wavelet transform (IDWT) for dealing with the watermarked image in R, G, B separate channel,

Step 6: Color images are combined into R, G, and B through channel combination approach. So the final watermarked image is obtained. According to the human eye sensitivity varies through different colors by R: G: B = 2:1:4.

\section{B. Watermark Extraction algorithm}

The extraction algorithm is the inverse of the embedding process shown in Fig. 3. Watermark is the seed value for authorized users at the receiver. The Watermark Extraction algorithm steps are as follows:



Fig. 3. Watermark extraction algorithm block diagram.

Step 1: Firstly in watermark extraction algorithm, the channel separation operation is applied on the watermarked color image and original image to generate its sub images.

Step 2: For the approximate coefficients and detail coefficients the 2-level discrete wavelet transform is applied on the sub images.

Step 3: After a DWT transform for the two-dimensional image, the watermark information is extracting from the watermarked image. According to the algorithm original color digital image is decomposed into three color components of $O(r), O(g), O(b)$, respectively.

Step 4: Finally original watermarked image decomposes into $W d(r), W d(g), W d(b)$ are three color components, respectively. In this process formula for the extraction algorithm is [12]:

$$
\begin{aligned}
& O(r) A(i, j)=O(r) A(i, j)+(w d(r) A(i, j)) / \alpha_{1} \\
& O(g) A(i, j)=O(g) A(i, j)+(w d(g) A(i, j)) / \alpha_{2} \\
& O(b) A(i, j)=O(b) A(i, j)+(w d(b) A(i, j)) / \alpha_{3}
\end{aligned}
$$

Step 5: Three watermark images (RGB) are extracted after the execution of above algorithm and application of inverse two levels Haar discrete wavelet transform.

Step 6: The original watermark image is obtained by combining the three $\mathrm{R}, \mathrm{G}$, and $\mathrm{B}$ watermark image.

\section{EXPERIMENT AND RESULTS}

The experiment is done with a PC having configuration of $2.4 \mathrm{GHz}$ personal laptop having 2GB RAM and Matlab 7.0 software platform is use to perform the experiment. The test set has been selected randomly for internet evaluation experiment watermark image. In the proposed algorithm experiment, we take $512 \times 512$ Lena and Girl as an example. The watermark is a visually recognizable color image which is 256×256 Ludong University logo. Fig. 4(a) and Fig. 5(a) shows the Original image and Fig. 4(b) and Fig. 5(b) shows Watermark image. Fig. 4(c) and Fig. 5(c) shows the watermarked image that is derived by embedding the watermark into the original image. Comparing Fig. 4(a), Fig. 5(a) and Fig. 4(c), Fig. 5(c), we can see that these two images look almost the same. Fig. 4(d) and Fig. 5(d) show the recovered watermark image from proposed methods. 


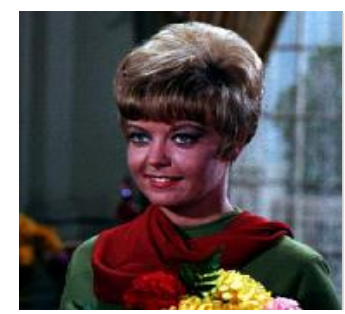

(a)



(c)

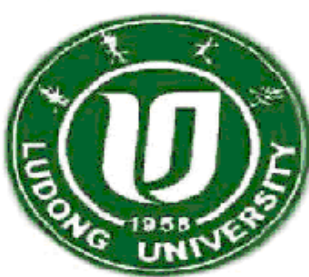

(b)

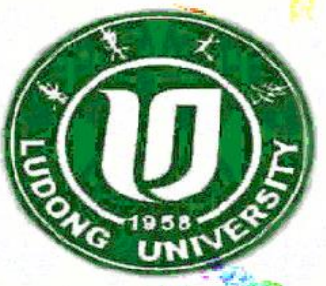

(d)
Fig. 4. (a) Original girl's image, (b) watermark image, (c) watermarked image, (d) recovered image.

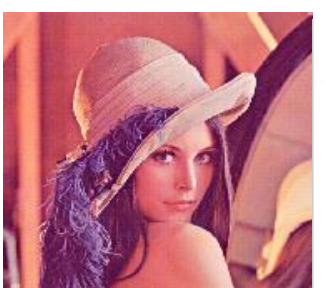

(a)

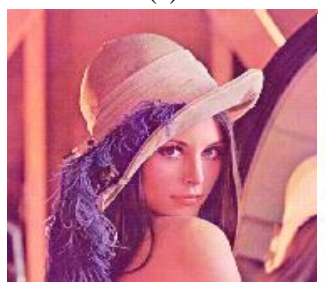

(c)

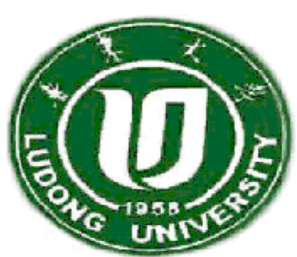

(b)

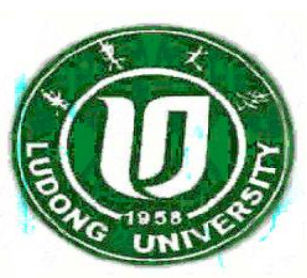

(d)
Fig. 5. (a) Original Lena image, (b) watermark image, (c) watermarked image, (d) recovered image.

In experiment of proposed algorithm for test the robustness, we experiments with the watermarked image by using common image processing operations such as adding salt and pepper noise, Gaussian noise, Brightness and contrast increment, Rotation on $45^{\circ}$ and $90^{\circ}$ and so on. The extracted images in Fig. 6 show the experimental results with these attacks. Experiment results show that the algorithm has robust when faced with attacks like salt and pepper noise, Gaussian noise, Brightness and contrast increment, Rotation.
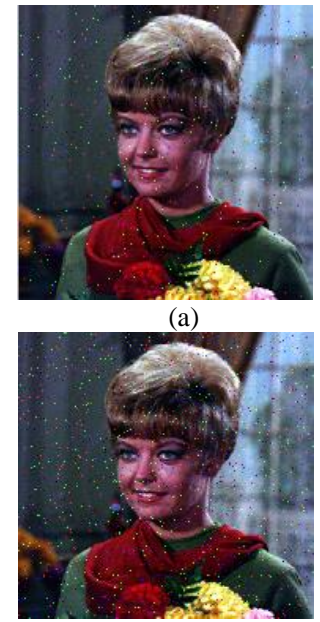

(c)

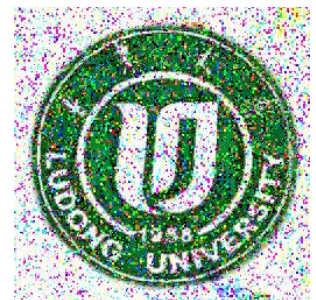

(b)

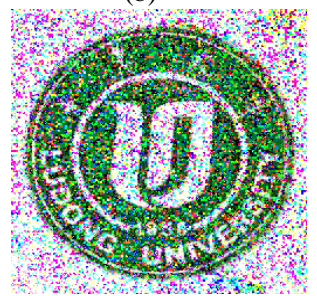

(d)

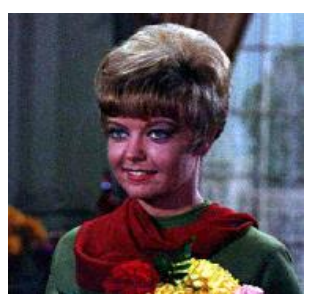

(e)

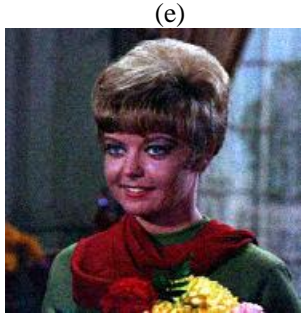

(g)



(i)
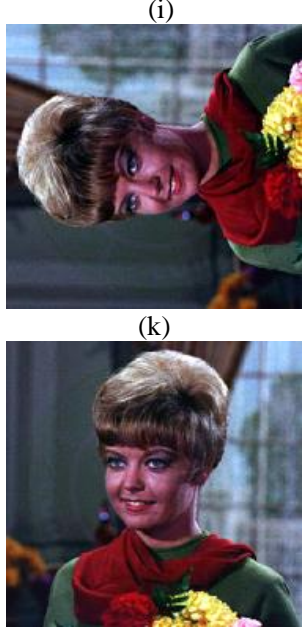

(m)

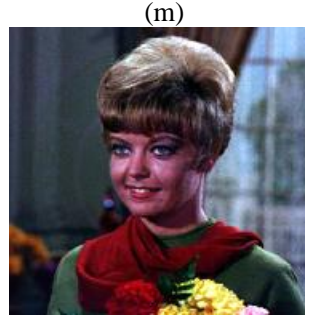

(o)



(f)



(h)

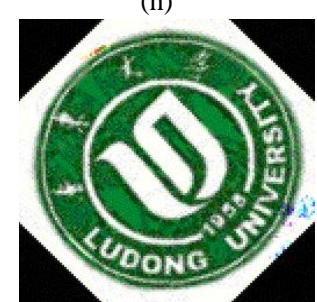

(j)



(1)

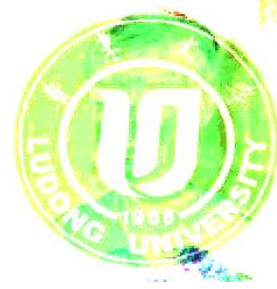

(n)

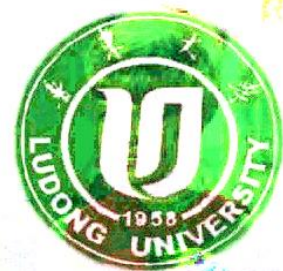

(p)

Fig. 6. (a) Pepper and salt noise(.01) watermarked, (b) recovered pepper and salt noise (.01) watermark, (c) pepper and salt noise(.02) watermarked, (d) recovered pepper and salt noise (.02) watermark, (e) Gaussian noise (.001) watermarked, (f) recovered Gaussian noise (.001) watermark, (g) Gaussian noise (.002) watermarked, (h) recovered Gaussian noise (.002) watermark, (i) rotation $45^{\circ}$ watermarked, (j) recovered rotation $45^{\circ}$ watermark, (k) rotation $90^{\circ}$ watermarked, (j) recovered rotation $90^{\circ}$ watermark, (m) brightness $(0.03,1)$ watermarked, (n) recovered brightness $(0.03,1)$ watermark, (o) contrast $(0.06,0.98)$ watermarked, $(\mathrm{p})$ recovered contrast $(0.06,0.98)$ watermark.

In this paper, the normalized correlation $N C$ between extracted watermark image $(\hat{W})$ and original watermark $(W)$ is used to evaluate the watermarking algorithm.

$$
N C=\frac{\sum_{i=1}^{M} \sum_{j=1}^{N} W(i, j) \hat{W}(i, j)}{\sum_{i=1}^{M} \sum_{j=1}^{M} W(i, j)^{2}}
$$


Compute the peak-signal-to-noise ratio (PSNR) from 8 , and multimedia. between the original image $X$ and the watermarked image $Y$.

$$
P S N R=10 \log \left(\frac{(M \times N)^{2}}{M S E}\right)
$$

where

$$
M S E=\frac{1}{M N} \sum_{i=1}^{M} \sum_{i=1}^{N}(Y(i, j)-X(i, j))^{2}
$$

The watermark extracted can be after identified even after attacks using results of Table I and Table II. This proves the robustness of the algorithm against of noisy, geometric and physical attacks.

TABLE I: EXPERIMENT RESULTS AND ANALYSIS ON GIRLS IMAGE

\begin{tabular}{|l|l|l|l|}
\hline \multirow{2}{*}{ Attack types } & Parameters & $\begin{array}{l}\text { Watermarked } \\
\text { PSNR/db }\end{array}$ & NC \\
\hline Original & Proposed & 36.691 & 0.9982 \\
\hline \multirow{2}{*}{$\begin{array}{c}\text { pepper-and-sal } \\
\text { t noise }\end{array}$} & 0.01 & 29.69 & 0.9312 \\
\cline { 2 - 4 } Gaussian noise & 0.02 & 27.22 & 0.8714 \\
\cline { 2 - 4 } & 0.001 & 33.415 & 0.9344 \\
\hline \multirow{2}{*}{ Rotation } & 0.002 & 31.610 & 0.8869 \\
\hline Brightness & $45^{\circ}$ & 38.410 & 0.6993 \\
\hline Contrast & $90^{\circ}$ & 36.690 & 0.8801 \\
\hline
\end{tabular}

TABLE II: EXPERIMENT RESULTS AND ANALYSIS ON LENA IMAGE

\begin{tabular}{|l|l|l|l|}
\hline Attack types & Parameters & $\begin{array}{l}\text { Watermarked } \\
\text { PSNR/db }\end{array}$ & NC \\
\hline Original & Proposed & 36.699 & 0.9897 \\
\hline \multirow{2}{*}{$\begin{array}{l}\text { pepper-and-sal } \\
\text { t noise }\end{array}$} & 0.01 & 30.176 & 0.9154 \\
\cline { 2 - 4 } Gaussian noise & 0.02 & 27.665 & 0.8502 \\
\cline { 2 - 4 } & 0.001 & 33.438 & 0.9122 \\
\hline \multirow{2}{*}{ Rotation } & 0.002 & 31.609 & 0.8580 \\
\hline Brightness & $45^{\circ}$ & 38.421 & 0.6952 \\
\hline Contrast & $(0.03,1)$ & 36.699 & 0.8705 \\
\hline
\end{tabular}

\section{CONCLUSION}

This paper gives a Nobel watermarking algorithm for color images based on discrete wavelet transform. The quality of the watermarked picture has a fine and enhanced nature of Peak signal to noise ratio. The proposed strategy is robust to loud, geometric and physical attacks. The experiment results are better as far as NC values of the extracted watermarks and PSNR of the watermarked image. At last the proposed algorithm is simple and has better in secure correspondence

\section{REFERENCES}

[1] S. Chen, B. Mulgrew, and P. M. Grant, "A clustering technique for digital communications channel equalization using radial basis function networks," IEEE Trans. on Neural Networks, vol. 4, pp. 570-578, July 1993.

[2] Q. Song and H. B. Zhang, "Color image self-embedding and watermarking based on DWT," in Proc. International Conference on Measuring Technology and Mechatronics Automation, 2010, vol. 1, pp. $796-799$.

[3] C. C. Lai and C. C. Tsai, "Digital image watermarking using discrete wavelet transform and singular value decomposition," IEEE Transactions on Instrumentation and Measurement, vol. 59, no. 11, pp. 3060-3063, 2010.

[4] J. Y. Bai and F. Liu, "A novel grayscale watermarking algorithm," in Proc. 2nd International Conference on Information Engineering and Computer Science, 2010, pp. 1-4.

[5] W. C. Chu, "DCT based image watermarking using sub sampling," IEEE Trans. on Multimedia, vol. 5, pp. 34-38, 2003.

[6] G. Sun and Y. Yu, "DWT based watermarking algorithm of color images," in Proc. 2nd IEEE Conference on Industrial Electronics and Applications, May 2007, pp. 1823-1826.

[7] P. S. Huang, C. S. Chiang, C. P. Chang, and T. M. Tu, "Robust spatial watermarking technique for colour images via direct saturation adjustment," Vision, Image and Signal Processing, vol. 152, issue 5, pp. 561-574, 2005.

[8] D. Kundur and D. Hatzinakos, "Towards robust logo watermarking using multiresolution image fusion," IEEE Transcations on Multimedia, vol. 6, issue 1, pp. 185-197, 2004.

[9] C. S. Lu and H. Y. M. Liao, "Multipurpose watermarking for image authentication and protection," IEEE Transaction on Image Processing, vol. 10, pp. 1579-1592, Oct. 2001.

[10] L. Ghouti, A. Bouridane, M. K. Ibrahim, and S. Boussakta, "Digital image watermarking using balanced multiwavelets," IEEE Trans. Signal Process., vol. 54, no. 4, pp. 1519-1536, 2006.

[11] P. Kumswat, K. Attakitmongcol, and A. Striaew, "A new approach for optimization in image watermarking by using genetic algorithms," IEEE Transactions on Signal Processing, vol. 53, no. 12, pp. 4707-4719, December 2005.

[12] Z. Zhou, Q. S. Ai, and Q. Liu, "Digital watermarking scheme for color image based on image fusion," in Proc. IET International Conference on Wireless, Mobile and Multimedia Networks, 2006, pp. 1-3.

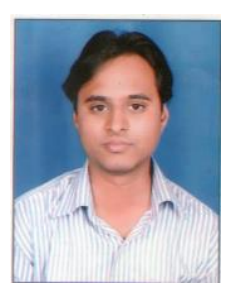

Anubhav Kumar was born in Bulandshahr, Uttar Pradesh, India on March 30, 1986. He received his B.Tech degree in electronics and communication engineering at Uttar Pradesh Technical University and M.Tech degree in electronics and communication engineering (specialization in image processing) at Uttar Pradesh Technical University. He is a member of IEEE, IACSIT and many international societies. He has also many years' research experiences in signal \& image processing and microwave engineering.

Anuradha received her B.Tech degree in electronics and communication engineering at Uttar Pradesh Technical University and now she is pursuing her M.Tech in VLSI design at Rajasthan Technical University. 